\title{
EL TRIBUNAL CONSTITUCIONAL ANTE UNA REFORMA SANITARIA APROBADA POR REAL DECRETO-LEY
}

\author{
Julio SÁNCHEZ FIERRO \\ Abogado \\ Vicepresidente de la Asociación Española \\ de Derecho Sanitario \\ jsfierro@delorenzoabogados.es
}

\section{CONSIDERACIONES GENERALES}

La esperada Sentencia del Tribunal Constitucional sobre la reforma sanitaria por fin vio la luz, despejando las dudas sobre la constitucionalidad de algunas medidas, ciertamente polémicas, contenidas en el Real Decreto-ley 16/2012, de 20 de abril. Se trata de la Sentencia 139/2016, de 21 de julio.

Conviene ante todo recordar la difícil coyuntura por la que atravesaba nuestro sistema sanitario público cuando se aprobó el mencionado Real Decreto-ley. El desajuste de las cuentas públicas sanitarias era tal que existían razones fundadas para preocuparse por la sostenibilidad de nuestro modelo sanitario. Una situación de acusada morosidad en los pagos a proveedores y más de 12.000 millones de euros de deuda acumulada están en la raíz misma del Real Decreto-ley.

Las medidas aprobadas fueron de muy diferente alcance, pero la asistencia sanitaria a inmigrantes irregulares y el nuevo régimen de copago farmacéutico han sido, sin duda, el centro del debate jurídico, político y social, y han concitado las mayores críticas.

En torno a la universalidad de la asistencia sanitaria, a los inmigrantes irregulares y al copago han girado los argumentos en pro y en contra de la reforma, dejando lamentablemente a un lado y para mejor ocasión otros problemas no menores de nuestro Sistema Nacional de Salud.

Me refiero a las crecientes desigualdades entre los pacientes en función del lugar en el que residan y al lamentable estado de «gobernanza» sanitaria con un consejo interterritorial que no acierta a cumplir con sus funciones de cohesión, de solidaridad y de coordinación entre los Servicios Autonómicos de Salud. 
El Real Decreto-ley 16/2012, de 20 de abril, de Medidas Urgentes para Garantizar la Sostenibilidad del Sistema Nacional de Salud y Mejora de la Calidad y Seguridad de sus Prestaciones, ha sido objeto de fuertes discrepancias en foros políticos e institucionales. Desafortunadamente los debates se han visto marcados más por la acritud de las posturas que por el rigor jurídico en los planteamientos.

Por eso mismo hay que considerar como un hecho muy positivo la claridad del fallo de nuestro más alto tribunal.

La Sentencia 139/2016, de 21 de julio, que resuelve el Recurso de Inconstitucionalidad 4123/2012 interpuesto por el Parlamento de Navarra, ha confirmado la constitucionalidad del referido Real Decreto-ley, ha fijado criterio en torno al principio de universalidad y ha dado su respaldo a la regulación sobre asistencia sanitaria a inmigrantes y, con algún matiz, al nuevo régimen jurídico aplicable al copago. A una y a otra materia nos referiremos a continuación.

\section{LA ASISTENCIA SANITARIA A INMIGRANTES IRREGULARES}

Valorando problemas, factores y circunstancias críticas que se registran, con mayor o menor intensidad, en los diversos Estados miembros, la Comisión Europea viene impulsando una política restrictiva para frenar la inmigración irregular, por entender que lo hace necesario la defensa de la frontera exterior comunitaria. De no tomar medidas para controlar el fenómeno inmigratorio, esta frontera común podría dejar de existir, porque resurgirían las fronteras entre los Estados miembros. Esto pondría en cuestión el propio proyecto comunitario.

Por ello, desde la Unión Europea se esta poniendo el acento en la aplicación de la normativa de expulsión, lo que, en materia sanitaria, implica propiciar una asistencia sanitaria de carácter temporal y estrictamente limitada a los casos de urgencia.

Ahora bien, hay que reconocer que esto no se ha traducido en una normativa uniforme en los países que integran la UE.

Ante este problemático contexto es preciso preguntarse cómo habrá que entender y aplicar el principio de asistencia sanitaria universal a los inmigrantes irregulares; un principio que tiene respaldo en normas internacionales (Pacto de Derechos Económicos, Sociales y Culturales; Carta Social Europea; Carta de Derechos Fundamentales de la UE; entre otros). 
En el caso de España esto supone determinar en qué medida el principio de universalidad puede o no conllevar la inaplicabilidad del Real Decreto-ley 16/2012, de Reforma de nuestro Sistema Nacional de Salud, que para el acceso a la asistencia sanitaria exige que el paciente tenga la condición de asegurado o de beneficiario en el marco del sistema de Seguridad Social.

Para poder pronunciarse, el punto de partida debería ser dilucidar cuáles son las características y el alcance del derecho a la protección de la salud consagrado por el art. 43 de nuestra Constitución.

Pues bien, lo primero que hay que tener presente es que este derecho, por su ubicación en el texto constitucional, no es un derecho fundamental, sino sólo un principio rector de la política social que, a tenor del art. 53 y concordantes, requiere ser objeto de «configuración» legal, esto es, para su efectividad necesita un desarrollo legal.

Esa configuración como derecho universal, hasta la fecha, no se ha producido, aunque, según la opinión más generalizada, la cobertura del sistema sanitario público se acerca al 99 por 100 de la población que reside en nuestro país.

Aunque, según algunos, la universalización del derecho a la protección de la salud estaría consagrada en la Ley General de Sanidad de 1986, lo cierto es que esta Ley hizo efectiva la asistencia sanitaria universal. La disposición transitoria quinta de dicha Ley anuncia que este derecho se aplicará «de modo progresivo».

La difícil situación económica que entonces vivía España (estábamos inmersos en el proceso de reconversión industrial) llevó al gobierno de entonces a esta «gradualización» o dosificación de la universalidad.

Semejante decisión se ha perpetuado durante años, tanto que en 2011 la Ley General de Salud Pública confirma este criterio y en su disposición adicional sexta vuelve a condicionar la universalidad a la «evolución de las cuentas públicas».

Esta falta de plenitud en el principio de universalidad llevó a los autores de la Ley General de Sanidad a establecer en su art. 16 la obligación que tienen las Administraciones sanitarias de facturar por la asistencia a quienes no tengan la condición de asegurado.

Otra manifestación de esa especie de «universalización parcial» se encuentra en la Ley 16/2003, de Cohesión y Calidad del Sistema Nacional de Salud, que proclamaba que: «Todos los españoles, así como los extranjeros con residencia legal, tienen derecho a la asistencia sanitaria». Por tanto, no lo tienen quienes estén en situación irregular. 
De otra parte, ¿ese «todos» significa acaso que ya ha dejado de ser necesaria la condición de «asegurado o beneficiario»? O lo que es igual, ¿la asistencia sanitaria ha quedado totalmente desvinculada del sistema de Seguridad Social?

Ciertamente, no. Baste recordar que el Pacto de Toledo ratificó en su día que las prestaciones sanitarias han de entenderse como prestaciones de Seguridad Social. En concreto, en el Pacto de Toledo se califican como prestaciones no contributivas y ello en razón al cambio de modelo de financiación de la sanidad: antes fue un modelo mixto (cotizaciones más impuestos) y a partir del Pacto de Financiación es un modelo sólo con impuestos.

Resulta inaceptable ignorar que en este planteamiento coincidieron todos los grupos parlamentarios firmantes del Pacto de Toledo.

De otra parte, el devenir jurídico no deja de ofrecernos algunas singulares peripecias. En efecto, en 2010 la Comisión Europea envió una carta de emplazamiento al gobierno español a propósito de la gestión de las prestaciones sanitarias en las Comunidades andaluza y valenciana.

Ambas Comunidades dejaron sin cobertura no a inmigrantes irregulares, sino a ciudadanos comunitarios residentes en Andalucía y Comunidad Valenciana, a pesar de cumplir con los requisitos de estar en posesión del TSE y no tener actividad económica.

El gobierno de entonces defendió la posición de las repetidas Comunidades, para lo cual no dudó en afirmar que, si bien la Seguridad Social es competencia del Estado, la gestión de las prestaciones sanitarias compete, según nuestra Constitución y Ley General de Sanidad, a las Comunidades Autónomas, y dado que esa gestión se financia con fondos autonómicos, las prestaciones han de considerarse asistencia social, la cual es competencia autonómica exclusiva a tenor del art. 148 CE.

En consecuencia, según el gobierno, el Reglamento comunitario 883/2004 (que modifica el Reglamento 1408/71, que opera en el marco de la Seguridad Social) no sería aplicable.

Sinceramente no creo que a día de hoy nadie se atrevería a defender que las prestaciones sanitarias comunes del Sistema Nacional de Salud son mera asistencia social.

Por supuesto, el Dictamen de la Comisión Europea rechazó de plano tan peregrina argumentación con fecha 24 de noviembre de 2011.

$\mathrm{El}$ «todos» de la Ley de Cohesión y Calidad ha supuesto un cambio del estatus legal de los inmigrantes irregulares, de modo que su situación ante la Seguridad Social ha pasado a ser algo irrelevante. 
La contestación ha de ser, en mi opinión, negativa, ya que si la Ley 4/2000, Orgánica de Extranjería, modificada por Ley 2/2009, mencionó sólo el requisito del empadronamiento, ello no supuso derogar el art. 14 de la misma, en el que, por cierto, se incardina el derecho a la asistencia sanitaria dentro del marco de la Seguridad Social.

Pues bien, refiriéndonos al art. 3 del tan citado Real Decretoley 16/2012, los extranjeros no registrados ni autorizados como residentes en España recibirán asistencia sanitaria en «situaciones de urgencia por enfermedad grave o accidente, cualquiera que sea su causa, hasta la situación de alta médica».

Asimismo, las mujeres tienen derecho a la «asistencia al embarazo, parto y posparto, y los mayores de dieciocho años con la misma extensión que los asegurados».

Esta cobertura estatal básica, comparable con otras de los diferentes Estados miembros de la UE, no es la única que tienen los inmigrantes irregulares. La Ley 33/2011, de 4 de octubre, General de Salud Pública, estableció para «toda la población», sin excepciones, acciones prevencionistas, asistenciales, de seguimiento y de control de situaciones de alerta y emergencia sanitaria.

Además, en el caso de los inmigrantes irregulares, éstos pueden completar la atención prevista en el RDL 16/2012 pidiendo a las Comunidades Autónomas su inclusión en los diferentes programas de asistencia social y sanitaria promovidos por ellas.

El Proyecto de Ley sobre sostenibilidad del Sistema Sanitario Público de Andalucía, actualmente en trámite, recoge y regula expresamente esta posibilidad.

En todo caso, la inclusión de los inmigrantes irregulares en los referidos programas puede tener como resultado darles acceso a una cobertura de similar alcance al establecido por la cartera común básica de servicios para los españoles, incluida la prestación farmacéutica que de ella se deriva, siempre que exista una indicación clínica por parte del correspondiente facultativo.

Es cierto que los programas autonómicos no son homogéneos a la hora de regular el contenido y los requisitos para el acceso a estas prestaciones (ver Anexo). Por ello, en su día el Ministerio de Sanidad propuso a las Comunidades Autónomas un acuerdo en el marco del Consejo Interterritorial del Sistema Nacional de Salud para homogeneizar la atención sanitaria de los inmigrantes irregulares. Desafortunadamente el acuerdo no llegó a ver la luz, de modo que este tema sigue en la lista de asuntos pendientes. 
Julio Sánchez Fierro $\quad$ El Tribunal Constitucional ante un reforma sanitaria aprobada...

Pienso que aún es tiempo para consensuar asuntos tales como:

- Modelo de solicitud.

- Forma de acreditar la identidad, la condición de mayor edad y de no tener derecho a la cobertura sanitaria por otra vía.

- Certificación de estar empadronado con una antigüedad de, en principio, seis meses y no disponer de recursos superiores al IPREM.

- Informe de circunstancias socioeconómicas elaborado por un mediador o trabajador social.

- Expedición de un documento acreditativo para acceder a estas prestaciones por órganos administrativos establecidos al efecto por la Comunidad Autónoma y con validez temporal.

Sin embargo, no sólo permanece aparcado un acuerdo como éste, que podría ser beneficioso y clarificador para los inmigrantes irregulares, sino que algunas Comunidades Autónomas pidieron y piden como premisa mayor la derogación del Real Decreto-ley 16/2012 y no han faltado otras que optaron por presentar un recurso de inconstitucionalidad, sobre el que ya ha recaído el correspondiente fallo y a cuyo contenido nos referiremos más adelante.

\section{EL COPAGO FARMACÉUTICO}

La participación del usuario en la financiación de los sistemas sanitarios públicos es una política común en los Estados miembros de la UE. Varía el régimen de copago según los países.

La regla general es que, bajo diversas modalidades, existan copagos para atención primaria, atención especializada y prestación farmacéutica, e incluso en algunos países (Portugal e Italia) en servicios de urgencia injustificados.

Hay estudios de distinto signo que describen esta realidad (por ejemplo, el de la fundación FEDEA).

Es un hecho que España, junto con Reino Unido y Dinamarca, no tienen establecidos copagos por asistencia sanitaria, aplicándolos sólo para la prestación farmacéutica.

A este respecto hay que decir en primer término que el copago farmacéutico siempre ha existido y que algunos que hoy reniegan de él lo defendieron con ahínco como «ticket moderador». 
En segundo lugar, es notorio que el Real Decreto de Servicios Comunes del SNS (RD 1030/2006) mantiene los copagos como regla general y que los pensionistas estaban exentos cualquiera que fuera su nivel de renta, mientras los funcionarios jubilados habían de hacer frente a un copago del 30 por 100, copago discriminatorio que fue muy contestado por estos pensionistas, pero, ciertamente, con poco éxito.

El Real Decreto-ley 16/2012 mantuvo el copago farmacéutico, pero introduciendo el factor nivel de renta e incluyendo a los pensionistas, salvo a los perceptores de las PNC.

El Real Decreto-ley, que introdujo algunas exenciones nuevas de evidente significado social (parados de larga duración), deslegalizó los tramos de renta (menos de 18.000 euros, entre 18.000 y 100.000 euros, y más 100.000 euros) para cuantificar el porcentaje de copago, los determinó por real decreto posterior y fijó unos topes mensuales de aportación $(8,18$ y 60 euros, según los casos).

Esta deslegalización ha sido valorada negativamente por el Tribunal Constitucional, según luego veremos.

En la actualidad ha resurgido la polémica sobre el copago farmacéutico. Veremos si se producen novedades o no.

\section{LA SENTENCIA DEL TRIBUNAL CONSTITUCIONAL}

El Real Decreto-ley 16/2012 fue recurrido y una importante sentencia del Tribunal Constitucional vino a confirmar que estaba plenamente ajustado a nuestra Carta Magna.

En efecto, la Sentencia núm. 139/2016, de 21 de julio, confirmó la constitucionalidad del referido Real Decreto-ley y con ella las medidas aprobadas por el gobierno. No obstante, como luego veremos, dos magistrados dejaron a salvo sus criterios discrepantes.

Con la Sentencia se aportan elementos que clarifican el concepto de universalidad y la valoración positiva de la urgencia para aprobar por decreto-ley medidas reformadoras para la sostenibilidad del sistema sanitario público, en particular las relativas a los extranjeros irregulares adultos y al copago farmacéutico, aunque esta última con un cierto reproche técnico (la remisión «en blanco» a vía reglamentaria para determinar el montante de ingresos a efectos de fijar el copago).

Seguidamente nos referiremos a los aspectos clave de la Sentencia: 
a) Se considera ajustado que se regule por Real Decreto-ley el alcance del derecho a la protección de la salud, ya que se trata de un principio rector cuyo desarrollo corresponde a los poderes públicos.

b) La regulación de las aportaciones de los usuarios no están vetadas a un Real Decreto-ley, ya que el copago no es necesariamente una «expresión» de la obligación de contribuir (propia de la ley).

c) No es cierto que el Real Decreto-ley afecte a la cartera de servicios complementaria, que es competencia de las Comunidades Autónomas.

d) El principio de universalidad no equivale a gratuidad de la asistencia sanitaria.

e) El derecho a la protección de la salud es un derecho de configuración legal, de modo que el cambio de criterio por parte del legislador no afecta a la constitucionalidad de las medidas.

f) Las medidas relativas a los inmigrantes no son arbitrarias, porque van dirigidas a la sostenibilidad del sistema sanitario público y, además, no se excluyen determinados supuestos de asistencia no urgentes, pero para ello se permite a la Administración sanitaria la posibilidad de exigir una contraprestación económica.

g) No se vulneran los tratados internacionales (art. $13 \mathrm{CE}$ ).

b) Los referidos tratados no son, per se, un canon para definir la constitucionalidad del Real Decreto-ley.

i) El Pacto de Derechos Económicos, Sociales y Culturales; la Carta de Derechos Fundamentales de la UE, y la Carta Social Europea no consagran el derecho de acceso gratuito y se remiten a las condiciones que al efecto fije el legislador nacional.

j) La deslegalización del montante de las aportaciones es criticable.

k) El Real Decreto-ley es fuente intercambiable con la ley en lo que se refiere a regular la aportación de los ciudadanos.

l) Se admite la posibilidad de copago en el caso de prestaciones sanitarias complementarias

m) El Real Decreto-ley no establece la cesión indebida de «datos tributarios». No cabe tal calificación, ya que sólo se trata de medir el nivel de aportación del usuario.

Por lo que se refiere a los votos particulares, éstos fueron presentados por los magistrados Fernando Valdés Dal-Re y Adela Asua. En esencia, ponen el acento en los siguientes motivos de discrepancia: 
a) No aprecian que concurra urgente necesidad para que se utilice la vía del Real Decreto-ley (a pesar de una situación de grave impago a proveedores y una deuda acumulada de 12.000 millones de euros).

b) Falta de conexión y de utilidad entre las medidas aprobadas y la vía de urgencia seguida por el gobierno.

c) Vulneración del art. 43 de la Constitución, que guarda conexión con el derecho a la vida e integridad física y moral (art. $15 \mathrm{CE}$ ), según declaró el ATC 239/2012.

d) Desproporción de las medidas.

g) No tener en cuenta el Convenio de Roma (art. 2) ni otras consideraciones doctrinales en torno al Pacto de Derechos Económicos, Sociales y Culturales.

\section{CONCLUSIONES}

A la vista de cuanto ha quedado expuesto cabe, en resumen, señalar lo siguiente:

1. La universalidad en la asistencia sanitaria es un objetivo establecido en la Ley General de Sanidad al que se dirige desde 1986 nuestro Sistema Nacional de Salud de modo gradual, estando próxima su efectividad plena.

2. La universalidad es un principio sujeto a configuración legal (articulación a través de la legislación ordinaria).

3. La universalidad no equivale sin más a la gratuidad en los servicios.

4. Los tratados internacionales no son un canon para evaluar la constitucionalidad del Real Decreto-ley y, por tanto, no sirven para definir el alcance y contenido del principio de universalidad.

5. Aunque, en principio, puede existir conexión conceptual entre el derecho a la protección de la salud y el derecho a la vida e integridad física, tal conexión no transforma la naturaleza del derecho a la protección de la salud (mero principio rector de política social) en un derecho fundamental. La insatisfacción que esto comporta explicaría que no falten quienes defienden una reforma constitucional para tutelar mejor el derecho a la protección de la salud, reconociéndole expresamente como derecho fundamental.

6. La atención sanitaria a los inmigrantes irregulares adultos puede llegar a ser muy similar a la de los españoles asegurados y bene- 
ficiarios sumando las prestaciones del art. 3 del Real Decreto-ley a las que pueden conceder las Comunidades Autónomas, bien como prestaciones complementarias de la cartera básica del Sistema Nacional de Salud, bien como parte de los programas asistenciales previstos en la Ley General de Salud Pública.

7. El objetivo de la universalidad no es incompatible con el modelo de asistencia sanitaria enmarcado dentro del sistema de Seguridad Social (asegurado/beneficiario). Basta con ver las páginas web de las Comunidades Autónomas, donde se recuerda el requisito de estar dado de alta en la Seguridad Social para obtener la tarjeta sanitaria.

8. El Real Decreto-ley 16/2012 no ha establecido un copago sanitario (por asistencia sanitaria), a diferencia de la mayoría de los países de nuestro entorno.

9. El Real Decreto-ley ha mantenido el copago farmacéutico, pero, como novedades, lo vincula al poder adquisitivo de los usuarios, elimina la exención de aportación de los pensionistas, salvo los perceptores de prestaciones no contributivas, y establece topes mensuales.

10. Algunas Comunidades Autónomas subvencionan el copago farmacéutico para evitar que éste sea una barrera de acceso a los medicamentos en el caso de los pacientes de menor poder adquisitivo.

11. La técnica jurídica utilizada en el Real Decreto-ley y en su desarrollo reglamentario para hacer operativo el copago farmacéutico (deslegalización y tramos de renta demasiado amplios) ha sido negativamente valorada por el TC y por diversos grupos políticos y sociales.

12. Se ha planteado la reconsideración del copago farmacéutico desde el propio gobierno, aunque no está claramente definido cuáles serían los cambios a introducir. 


\author{
ANEXO \\ SITUACIÓN DE LA ATENCIÓN SANITARIA \\ A INMIGRANTES IRREGULARES POR COMUNIDADES \\ AUTÓNOMAS SEGÚN LOS RESPECTIVOS \\ GOBIERNOS AUTONÓMICOS
}

\begin{abstract}
ANDALUCÍA
La Junta de Andalucía se opuso a la exclusión de los irregulares y siguió prestando asistencia sanitaria a las personas extranjeras que no tienen regularizada su residencia en la Comunidad y que carecen de recursos económicos y aseguramiento. Este derecho, que se viene garantizando desde 1999, «es una decisión solidaria que intenta proteger a la población más vulnerable y sin recursos».

En esta Comunidad, la población inmigrante recibe un documento de reconocimiento temporal que se emite en los distritos de atención primaria y que garantiza el acceso al sistema sanitario público dentro de Andalucía. Con este documento se hace posible la identificación de las personas inmigrantes y permite hacer un seguimiento de los servicios que se les presta y de su estado de salud.

Desde que en 2012 entrara en vigor el Real Decreto del gobierno que deja sin cobertura a las personas inmigrantes, Andalucía ha reconocido el derecho a la asistencia sanitaria a más de 110.000 personas inmigrantes que se hubieran quedado sin prestación sanitaria con la normativa estatal. Actualmente son 27.000 los inmigrantes que tienen documento sanitario temporal.
\end{abstract}

\title{
ARAGÓN
}

Aragón, desde el pasado 13 de agosto, también se encaminó hacia la asistencia sanitaria universal para todos los ciudadanos de la Comunidad. El Departamento de Sanidad de la Junta trasladó a todos los centros de atención primaria y especializada las instrucciones para extender la cobertura sanitaria en la región de manera inmediata.

Las condiciones para acceder a la cobertura total del Servicio Aragonés de Salud son que los beneficiarios lleven al menos tres meses empadrona- 
dos en la Comunidad y que no tengan condición de asegurado por ninguna otra Administración o que no puedan exportar el derecho de asistencia sanitaria desde su país de origen. Además, sus ingresos deben ser inferiores en dos veces y media el IPREM anual (menos de 16.000 euros anuales). La duración es de doce meses prorrogables, si se mantienen las condiciones.

\section{ASTURIAS}

Asturias mostró desde el primer momento su oposición al Real Decreto-ley 16/2012 y anunció que atendería a los inmigrantes irregulares a través del servicio público de salud aunque carecieran de tarjeta, incluidas consultas con especialistas.

Se estableció así un procedimiento administrativo para inscribir a las personas extranjeras sin recursos económicos empadronadas en Asturias en el Sistema de Información de Población y Recursos Sanitarios (SIPRES).

Este procedimiento contempla que toda persona extranjera sin recursos que resida en el Principado y lo solicite, independientemente de su situación administrativa, se incorpore a la base de datos para que se le asigne un centro de salud y un médico de referencia, de manera que se le garantice el acceso a los servicios sanitarios autonómicos. En la actualidad hay 1.368 personas dadas de alta en el SIPRES como extranjeros sin recursos en situación administrativa irregular.

Además, el Servicio de Salud articuló en colaboración con Cruz Roja un procedimiento para permitir a estos colectivos acceder a la prestación farmacéutica en condiciones semejantes a la población general.

\section{BALEARES}

En Baleares, el nuevo ejecutivo incluyó en el sistema de salud pública balear el pasado 17 de julio a unos 15.000 extranjeros que residen irregularmente en las islas. Estos tendrán una tarjeta identificativa con la que podrán recibir asistencia médica y farmacéutica de manera gratuita en el sistema de salud pública.

Ahora, los inmigrantes que soliciten ser incluidos en el sistema público deberán efectuar una declaración jurada sobre su situación y falta de recursos. Serán integrados en el sistema informático sanitario y tendrán 
Julio Sánchez Fierro El Tribunal Constitucional ante un reforma sanitaria aprobada...

un documento válido para la Comunidad de Baleares durante un año, que será renovable.

\section{CANARIAS}

La nueva regulación de 2013 para los inmigrantes en Canarias tiene importantes limitaciones. La primera, que cierra los centros sanitarios a los que no puedan acreditar que ganan menos de 532 euros al mes.

Sanidad considera que a partir de esa cantidad la necesidad de cobertura sanitaria gratuita no es tan perentoria.

La segunda es el tiempo que llevan residiendo en Canarias. Para poder asistir al médico tendrán que demostrar que han estado empadronados, aunque sea en distintos municipios canarios, desde hace más de un año.

El nuevo gobierno de Canarias ha declarado que garantizará «muy pronto» la universalidad de la sanidad al cubrir la asistencia médica a todos los inmigrantes que se encuentran en las islas de forma irregular.

\section{CANTABRIA}

Cantabria prestará asistencia sanitaria a inmigrantes irregulares que lleven tres meses empadronados en la Comunidad Autónoma e independientemente de sus ingresos económicos.

\section{CASTILLA-LA MANCHA}

Según la Consejería de Sanidad, se está trabajando para ampliar la normativa que permitirá atender a los irregulares.

\section{CASTILLA Y LEÓN}

El Servicio de Salud de Castilla y León (SACYL) fue tajante desde un principio: atendería en sus instalaciones a todos los inmigrantes.

La Junta estima que de esta decisión se benefician entre 4.500 y 5.000 inmigrantes en situación irregular. 
Julio Sánchez Fierro $\quad$ El Tribunal Constitucional ante un reforma sanitaria aprobada...

\section{CATALUÑA}

Los inmigrantes no tendrán que esperar doce meses para tener vía libre a todos los servicios sanitarios públicos.

Una nueva instrucción del departamento de salud que entró en vigor el pasado 23 de julio autoriza el acceso a la totalidad de la cartera básica de servicios (urgencias, primaria y especialistas) a todos los ciudadanos extranjeros que acrediten un empadronamiento de tres meses.

Los inmigrantes que cumplan ese requisito recibirán una tarjeta sanitaria «similar» a la oficial, que deberán renovar transcurrido un año y que les garantiza cobertura sanitaria completa en la red catalana.

Pasarán a tener cobertura plena 70.000 inmigrantes en situación irregular.

\section{EXTREMADURA}

La Consejería de Sanidad anunció que aprobará una norma para que la prestación sanitaria a los inmigrantes sin papeles «tenga realmente carácter universal».

Desde esta Consejería se recuerda que en Extremadura los inmigrantes sin papeles son atendidos con normalidad y tienen derecho a la prestación universal los menores y los que son atendidos en urgencias. Además, el resto dispone de un documento que les da derecho a la atención sanitaria y al 50 por 100 de las prestación farmacéutica a través de ONGs —otro 50 por 100 corre a su cargo-.

\section{GALICIA}

Los «sin papeles» sólo tienen que estar censados para recibir atención médica tanto en los centros sanitarios como en los hospitales.

La decisión fue adoptada en 2012 y mantiene la asistencia sanitaria a este colectivo e incluso les concede una tarjeta de iguales características que la del resto de usuarios.

Los requisitos para lograrla son dos. El primero, estar empadronados en la localidad donde residan y contar con un tiempo mínimo de estancia en ella que fue fijado en 183 días. El segundo, no disponer de ingresos supe- 
riores al Indicador Público de Renta de Efectos Múltiples (IPREM) ni en España ni en su país de origen, una cantidad que ronda los 535 euros al mes.

Los inmigrantes ilegales que cumplen con estos dos requerimientos no cuentan con limitaciones en el contenido sanitario, de forma que reciben asistencia en la atención primaria y también en la especializada.

Se calcula que en Galicia hay unas 9.000 personas en esta situación.

\section{LA RIOJA}

El gobierno riojano ha aplicado el Real Decreto-ley tal y como lo estableció el gobierno central. El Sistema Público de Salud de La Rioja presta asistencia sanitaria a todos los extranjeros irregulares que lo piden y, posteriormente, emite la correspondiente factura para cobrarles esa prestación, con excepción de menores, mujeres embarazadas y en casos urgentes.

\section{COMUNIDAD DE MADRID}

La Comunidad de Madrid añadió algunos supuestos en los que los inmigrantes tienen también derecho a atención: los solicitantes de asilo y las víctimas de trata cuya estancia temporal en España haya sido autoriza$\mathrm{da}$; los pacientes de enfermedades infectocontagiosas, y las personas con enfermedades crónicas cuyo tratamiento se haya iniciado antes del 31 de agosto de 2012.

Está previsto crear un documento específico - $\mathrm{y}$ «diferente» de la tradicional tarjeta - para dar cobertura completa a los inmigrantes en situación irregular, que podrán acceder a la atención primaria y especializada dentro de la región.

Además, se ha ordenado - mediante el envío de una circular - a todos los gerentes de hospitales y directores de centros de salud de la región prestar atención sanitaria a los inmigrantes «con o sin documentación» de forma inmediata.

\section{NAVARRA}

El gobierno navarro cuenta desde 2013 con una legislación garantista de la salud como derecho universal. La Ley Navarra de Asistencia Sani- 
taria Gratuita reconoce a los residentes en la Comunidad Foral, incluidos extranjeros y residentes en situación irregular, el derecho a la asistencia sanitaria gratuita pública primaria o especializada.

Se entiende por «residencia» el hecho de acreditar, por cualquier medio admitido en Derecho, incluido el empadronamiento, que el domicilio de la persona está en un municipio de Navarra, sin atención a tiempo alguno.

Aportada la documentación que acredite la residencia, la Administración sanitaria expide, gratuitamente y sin dilación, un documento de identificación de acceso al régimen de universalización de la asistencia sanitaria.

\section{PAÍS VASCO}

El Servicio Vasco de Salud comenzó a distribuir en noviembre de 2014 una Tarjeta Individual Sanitaria (TIS), sólo válida en la Comunidad, a los alrededor de 17.000 inmigrantes en situación irregular que viven en la región. Los requisitos son acreditar un año de empadronamiento continuado y tener unos ingresos inferiores a la Renta de Garantía de Ingresos (RGI).

En el caso de las mujeres embarazadas que lleven menos tiempo viviendo en Euskadi, tendrán el mismo derecho durante el embarazo, parto y posparto, así como los menores de dieciocho años.

La regulación establece que el resto de inmigrantes, es decir, los que no lleven un año empadronados o cobren por encima de la RGI, son atendidos en Osakidetza de manera gratuita cuando se traten de urgencias y de problemas de salud. En los demás supuestos, los pacientes deben hacerse cargo del coste de su atención.

\section{REGIÓN DE MURCIA}

La Consejería de Sanidad ha anunciado que todos los inmigrantes «sin papeles» en la región volverán a tener asistencia sanitaria «completa».

Fuentes de la Consejería han aclarado que lo que está en estudio es ofrecer a los «sin papeles» algún tipo de documento que les permitía acceder al sistema sin que ello suponga la devolución de la tarjeta sanitaria. 


\section{COMUNIDAD VALENCIANA}

La Generalitat Valenciana fue el primer gobierno salido de las urnas de las últimas elecciones de mayo que anunció la extensión de la asistencia sanitaria a todos los empadronados en su territorio.

Cerca de 30.000 inmigrantes irregulares que lleven al menos tres meses empadronados son los beneficiarios de una de las últimas medidas que ha tomado la Consellería de Sanidad.

A finales de julio se comenzó a expedir el SIP tras la entrada en vigor del Decreto-ley 3/2015 que daba «respuesta a la situación de indefensión sanitaria» en la que se encontraban las «personas extranjeras en situación administrativa irregular y socialmente desfavorecida»

Tener nacionalidad extranjera, ser mayor de edad y no tener cobertura sanitaria en ningún otro país son los tres requisitos necesarios para la expedición de la tarjeta sanitaria, junto al precepto de estar empadronado en la Comunidad al menos tres meses antes de la solicitud del SIP. La cobertura incorpora, además de la cartera común básica de servicios, la asistencia especializada en hospital y su tratamiento farmacológico.

También incluye la prescripción de medicamentos y prestaciones ortoprotésicas. 
